\title{
THE RESTRICTED WEIGHTED GENERALIZED INVERSE OF A MATRIX*
}

\author{
VASILIOS N. KATSIKIS ${ }^{\dagger}$ AND DIMITRIOS PAPPAS $\ddagger$
}

\begin{abstract}
We introduce the $T$-restricted weighted generalized inverse of a singular matrix $A$ with respect to a positive semidefinite matrix $T$, which defines a seminorm for the space. The new approach proposed is that since $T$ is positive semidefinite, the minimal seminorm solution is considered for all vectors perpendicular to the kernel of $T$.
\end{abstract}

Key words. Moore-Penrose inverse, Weighted Moore-Penrose inverse.

AMS subject classifications. 15A09.

1. Introduction. Quadratic forms have played a central role in the history of mathematics in both the finite and infinite dimensional cases. A number of authors have studied problems on minimizing (or maximizing) quadratic forms under various constraints such as vectors constrained to lie within the unit simplex (Broom [2]), and the minimization of a more general case of a quadratic form defined in a finite-dimensional real Euclidean space under linear constraints (see, e.g., La Cruz [5], Manherz and Hakimi [9]), with many applications in network analysis and control theory (for more on this subject, see also $[16,17])$. In his classical book on optimization theory, Luenberger [13], presents similar optimization problems for both finite and infinite dimensions.

In the field of applied mathematics, one sees an interest in applications of the generalized inverse of matrices or operators (see [1]). In many computational and theoretical problems, whenever a matrix is singular, various types of generalized inverses are used. An important application of the Moore-Penrose inverse in the finite dimensional case is the minimization of a hermitian positive definite quadratic form $x^{\prime} T x$ under linear constraints. In this article, we propose another approach for the case of a positive semidefinite quadratic form by choosing the constrained minimization problem to take place only for the vectors perpendicular to its kernel.

*Received by the editors on July 5, 2011. Accepted for publication on November 20, 2011. Handling Editor: Michael Tsatsomeros.

${ }^{\dagger}$ General Department of Mathematics, Technological Education Institute of Piraeus, Aigaleo, 12244 Athens, Greece (vaskats@gmail.com).

$¥$ Athens University of Economics and Business, Department of Statistics, 76 Patission Str, 10434, Athens, Greece (dpappas@aueb.gr, pappdimitris@gmail.com). 
The material of this article provides the opportunity for research in several different directions. In particular, one useful financial application is based on the Capital Asset Pricing Model (CAPM) and the Arbitrage Pricing Theory (APT) models. The CAPM is a variation of the classical portfolio selection problem, as defined by Markowitz (1952) [14]. It uses the minimization of a quadratic form $x^{\prime} \Sigma x$ under linear constraints that can be written in matrix form $A x=b$, where $\Sigma$ is the positive semidefinite variance-covariance matrix of the assets. In fact, $\Sigma$ is usually positive definite, but cases exist where $\Sigma$ is singular. Since the solution to this problem needs the inverse matrix $\Sigma^{-1}$, there is a clear need for developing algorithms that deal with the singular case. APT models describe another influential theory on asset pricing. They differ from the CAPM in that they are less restrictive in their assumptions allowing for an explanatory (as opposed to statistical) model of asset returns. Their implementation also requires inversion of a possibly singular covariance matrix; see, for example, [3] which is a standard reference book on financial applications.

There are five sections following this section of introduction. Section 2 is a quick review of the fundamental properties of generalized inverses. In Section 3, the theoretical background for the restricted weighted generalized inverse is discussed together with the main results of this work. Relations with the $V$-orthogonal projector, as described in [21], are presented in Section 4. In Section 5, we test the efficiency of the proposed method. For the exhibition of the effectiveness of our proposed method, we have performed numerical experiments for the proposed constrained minimization problem for both full and sparse positive semidefinite matrices. In particular, Section 5 is divided in two subsections: The first subsection gives numerical results of the proposed method for the case of non-sparse positive semidefinite matrices while the second one gives the corresponding results for the case of sparse positive semidefinite matrices. Conclusions are provided in Section 6 .

2. Preliminaries and notation. From now on $\mathcal{H}$ will denote a finite dimensional Hilbert space (e.g., $\mathbb{R}^{n}$ or $\mathbb{C}^{n}$ ) and $\mathcal{B}(\mathcal{H})$ will denote the set of matrices acting on $\mathcal{H}$. The results of this paper can also be extended to infinite dimensional Hilbert spaces and operators instead of matrices.

The notion of the generalized inverse of a matrix was first introduced by H. Moore in 1920 and again by R. Penrose in 1955. These two definitions are equivalent and the generalized inverse of an operator or matrix is also called the Moore-Penrose inverse. In the case when $A$ is a real $r \times m$ matrix, Penrose showed that there is a unique matrix satisfying the four Penrose equations, called the generalized inverse of $A$ and denoted by $A^{\dagger}$ :

$$
A A^{\dagger}=\left(A A^{\dagger}\right)^{*}, \quad A^{\dagger} A=\left(A^{\dagger} A\right)^{*}, \quad A A^{\dagger} A=A, \quad A^{\dagger} A A^{\dagger}=A^{\dagger},
$$

where $A^{*}$ denotes the conjugate transpose of $A$. 
It is easy to see that $A A^{\dagger}$ is the orthogonal projection of $\mathcal{H}$ onto the range $\mathcal{R}(A)$ of $A$, denoted by $P_{A}$, and that $A^{\dagger} A$ is the orthogonal projection of $\mathcal{H}$ onto $\mathcal{R}\left(A^{*}\right)$ denoted by $P_{A^{*}}$. It is also well known that $\mathcal{R}\left(A^{\dagger}\right)=\mathcal{R}\left(A^{*}\right)$.

Let us consider the equation $A x=b, A \in B(\mathcal{H})$, where $A$ is singular. If $b \notin \mathcal{R}(A)$, then the equation has no solution. In this case we consider the equation $A x=P_{\mathcal{R}(A)} b$, where $P_{\mathcal{R}(A)}$ is the orthogonal projection on $\mathcal{R}(A)$.

The following two propositions can be found in Groetsch [8, Theorem 2.1.1 and Definition (V), pages 39 and 41].

Proposition 2.1. Let $A \in \mathcal{B}(\mathcal{H})$ and $b \in \mathcal{H}$. Then, for $u \in \mathcal{H}$, the following are equivalent:

(i) $A u=P_{\mathcal{R}(T)} b$.

(ii) $\|A u-b\| \leq\|A x-b\|, \forall x \in \mathcal{H}$.

(iii) $A^{*} A u=A^{*} b$

Let $\mathbb{B}=\left\{u \in \mathcal{H} \mid A^{*} A u=A^{*} b\right\}$. Then this set $\mathbb{B}$ is closed and convex; it therefore has a unique vector with minimal norm. In the literature, (e.g., Groetsch [8, page $41]), \mathbb{B}$ is known as the set of the generalized solutions.

Proposition 2.2. For $A \in \mathcal{B}(\mathcal{H})$ and $b \in \mathcal{H}$, consider the equation $A x=b$. Then it holds $A^{\dagger} b=u$, where $u$ is the minimal norm solution in $\mathbb{B}$.

This property has an application in the problem of minimizing a hermitian positive definite quadratic form $\langle x, Q x\rangle$ subject to linear constraints which are assumed to be consistent.

3. The restricted weighted generalized inverse. For this section, we need the notion of the weighted Moore-Penrose inverse of a matrix $A \in \mathbb{C}^{m \times n}$ with respect to two Hermitian positive definite matrices $M \in \mathbb{C}^{m \times m}$ and $N \in \mathbb{C}^{n \times n}$ denoted by $X=A_{M, N}^{\dagger}$ satisfying the following four equations (see [4, page 118, Exercise 30] or [22, Section 3]; for computational methods, see [20], and for more on this subject, see $[6,7])$ :

$$
A X A=A, \quad X A X=X
$$

$$
(M A X)^{*}=M A X
$$

$$
(N X A)^{*}=N X A
$$

It is also known (see, e.g., [1]) that

$$
A_{M, N}^{\dagger}=N^{-\frac{1}{2}}\left(M^{\frac{1}{2}} A N^{-\frac{1}{2}}\right)^{\dagger} M^{\frac{1}{2}}
$$

In this case, $A_{M, N}^{\dagger} b$ is the $M$-least squares solution of $A x=b$ which has minimal $N$-norm.

This notion can be extended to the case for which $M$ and $N$ are positive semidefinite matrices. In this case, $G$ is a matrix such that $G b$ is a minimal $N$ semi-norm, 
$M$-least squares solution of $A x=b$. Subsequently, $G$ must satisfy the following four conditions (See [4], page 118, exercises 31-34):

(3.2) $M A G A=M A, \quad N G A G=N G, \quad(M A G)^{*}=M A G, \quad(N G A)^{*}=N G A$.

When $N$ is positive definite, then there exists a unique solution for $G$.

As mentioned above, the Moore-Penrose inverse has an application in minimizing a constrained quadratic form. As presented in Pappas [19], we may look for the minimum of a positive semidefinite quadratic form $\langle x, T x\rangle$ among the vectors $x \in$ $\mathcal{N}(T)^{\perp}=\mathcal{R}\left(T^{*}\right)=\mathcal{R}(T)$, or equivalently, under the constraints $A x=b, x \in \mathcal{R}(T)$. The following theorem holds for linear bounded operators acting on an infinite dimensional Hilbert space $\mathcal{H}, T$ denotes a singular positive operator with a canonical form $T=U\left(T_{1} \oplus 0\right) U^{*}$ where $U$ is a unitary operator, $R$ being the unique positive solution of the equation $R^{2}=T_{1}$ and $R^{\dagger}=\left[\begin{array}{cc}R^{-1} & 0 \\ 0 & 0\end{array}\right]$. (For more on the canonical form of singular hermitian matrices and operators, see [4, Chapter 4].)

TheOREM 3.1. ([19, Theorem 3.9]) Let $T \in \mathcal{B}(\mathcal{H})$ be a singular positive operator, and the equation $A x=b$, with $A \in \mathcal{B}(\mathcal{H})$ singular with closed range and $b \in \mathcal{H}$. If the set $S=\left\{x \in \mathcal{N}(T)^{\perp}: A x=b\right\}$ is not empty, then the problem:

$$
\operatorname{minimize}\langle x, T x\rangle, x \in S
$$

has the unique solution

$$
\hat{x}=U R^{\dagger}\left(A U R^{\dagger}\right)^{\dagger} b
$$

assuming that $P_{A^{*}} P_{T}$ has closed range.

By rephrasing Theorem 3.1 for the finite dimensional case, while taking into account the fact that for unitary matrices, we have $U^{*}=U^{\dagger}$ and that $U R^{\dagger} U^{*}=$ $\left(T^{\dagger}\right)^{\frac{1}{2}}$, we deduce the following theorem.

THEOREM 3.2. Let $T \in \mathbb{R}^{m \times m}$ be a positive semidefinite hermitian matrix, and the equation $A x=b$ with $A \in \mathbb{R}^{n \times m}$ and $b \in \mathbb{R}^{m}$. If the set $S=\left\{x \in \mathcal{N}(T)^{\perp}: A x=\right.$ b) is not empty, then the problem:

$$
\operatorname{minimize}\langle x, T x\rangle, x \in S
$$

has the unique least squares solution

$$
\hat{u}=\left(T^{\dagger}\right)^{\frac{1}{2}}\left(A\left(T^{\dagger}\right)^{\frac{1}{2}}\right)^{\dagger} b .
$$


Based on Theorem 3.2, similarly as the weighted Moore-Penrose inverse, we can extend this notion to the $N$-restricted weighted inverse with $M$ positive definite and $N$ a positive semidefinite matrix:

$$
\left.\hat{A}_{M, N}^{\dagger}=\left(N^{\dagger}\right)^{\frac{1}{2}}\left(M^{\frac{1}{2}} A\left(N^{\dagger}\right)^{\frac{1}{2}}\right)^{\dagger}\right)^{\dagger} M^{\frac{1}{2}}
$$

giving a solution such that $\hat{A}_{M, N}^{\dagger} b$ is a minimal $N$ semi- norm, $M$-least squares solution of $A x=b$ but restricted on the range of $N$.

Definition 3.3. Let $T \in \mathbb{R}^{m \times m}$ be a positive semidefinite hermitian matrix and $A \in \mathbb{R}^{n \times m}$. Then the $m \times n$ matrix

$$
\hat{A}_{I_{n}, T}^{\dagger}=\left(T^{\dagger}\right)^{\frac{1}{2}}\left(A\left(T^{\dagger}\right)^{\frac{1}{2}}\right)^{\dagger}
$$

is the $T$-restricted weighted Moore-Penrose inverse of $A$, such that $\hat{A}_{I_{n}, T}^{\dagger} b$ is a minimal $T$ semi-norm least squares solution of $A x=b$, restricted on the range of $T$.

Using the above definition, the solution becomes

$$
\hat{u}=\hat{A}_{I_{n}, T}^{\dagger} b
$$

and since $T$ is positive semidefinite, $\langle x, T x\rangle$ defines a seminorm for the space $\mathbb{C}^{n}$. Therefore, $\hat{u}$ is a minimal $T$ semi-norm least squares solution of $A x=b$.

We can verify that the solution $\hat{u}$ satisfies the constraint $A x=b$. Indeed, $A \hat{u}=$ $A\left(T^{\dagger}\right)^{\frac{1}{2}}\left(A\left(T^{\dagger}\right)^{\frac{1}{2}}\right)^{\dagger} b=P_{A T} b$, and since the set $S=\{x \in \mathcal{R}(T): A x=b\}$ is not empty, we have that $b$ must be equal to $A T w$ for some $w$ and therefore $P_{A T} b=b$.

We can also notice that the matrix $\hat{A}_{I_{n}, T}^{\dagger}$ does not satisfy all four conditions of equation (3.2) as it is an inverse restricted to the range of $T$. Similarly to the equations (3.2) we have the following:

Proposition 3.4. Let $T \in \mathbb{R}^{m \times m}$ be positive semidefinite, $A \in \mathbb{R}^{n \times m}$ and the equation $A x=b$. Then the T-restricted weighted inverse $\hat{A}_{I, T}^{\dagger}$ satisfies the following conditions:

(i) $A \hat{A}_{I, T}^{\dagger} A=P_{A T} A$.

(ii) $T \hat{A}_{I, T}^{\dagger} A \hat{A}_{I, T}^{\dagger}=T \hat{A}_{I, T}^{\dagger}$.

(iii) $\left(A \hat{A}_{I, T}^{\dagger}\right)^{*}=\left(A \hat{A}_{I, T}^{\dagger}\right)$.

(iv) $\hat{A}_{I, T}^{\dagger} A \hat{A}_{I, T}^{\dagger}=\hat{A}_{I, T}^{\dagger} P_{A T}$.

Proof.

(i) $A \hat{A}_{I, T}^{\dagger} A=A\left(T^{\dagger}\right)^{\frac{1}{2}}\left(A\left(T^{\dagger}\right)^{\frac{1}{2}}\right)^{\dagger} A=P_{A T^{\dagger}} A=P_{A T} A$.

(ii) $T \hat{A}_{I, T}^{\dagger} A \hat{A}_{I, T}^{\dagger}=T\left(T^{\dagger}\right)^{\frac{1}{2}}\left(A\left(T^{\dagger}\right)^{\frac{1}{2}}\right)^{\dagger} A\left(T^{\dagger}\right)^{\frac{1}{2}}\left(A\left(T^{\dagger}\right)^{\frac{1}{2}}\right)^{\dagger}=T\left(T^{\dagger}\right)^{\frac{1}{2}}\left(A\left(T^{\dagger}\right)^{\frac{1}{2}}\right)^{\dagger}=$ $T \hat{A}_{I, T}^{\dagger}$. 
(iii) $\left(A \hat{A}_{I, T}^{\dagger}\right)^{*}=\left(A\left(T^{\dagger}\right)^{\frac{1}{2}}\left(A\left(T^{\dagger}\right)^{\frac{1}{2}}\right)^{\dagger}\right)^{*}=\left(P_{A T^{\dagger}}\right)^{*}=P_{A T^{\dagger}}=A \hat{A}_{I, T}^{\dagger}$.

(iv) $\hat{A}_{I, T}^{\dagger} A \hat{A}_{I, T}^{\dagger}=\left(T^{\dagger}\right)^{\frac{1}{2}}\left(A\left(T^{\dagger}\right)^{\frac{1}{2}}\right)^{\dagger} A\left(T^{\dagger}\right)^{\frac{1}{2}}\left(A\left(T^{\dagger}\right)^{\frac{1}{2}}\right)^{\dagger}=\left(T^{\dagger}\right)^{\frac{1}{2}}\left(A\left(T^{\dagger}\right)^{\frac{1}{2}}\right)^{\dagger} P_{A T^{\frac{1}{2}}}=$ $\hat{A}_{I, T}^{\dagger} P_{A T}$.

From the above conditions it is clear that $\hat{A}_{I, T}^{\dagger}$ is not an $\{i, j, k\}$ inverse of $A$. Nevertheless, many of the known properties of the generalized inverses also hold for the $T$-restricted weighted inverse, with slight modifications, as we can see in the following proposition.

Proposition 3.5. Let $T \in \mathbb{R}^{m \times m}$ be positive semidefinite and $A \in \mathbb{R}^{n \times m}$. The T-restricted weighted inverse $\hat{A}_{I, T}^{\dagger}$ has the following properties:

(i) If $\hat{A}_{I, T}^{\dagger}=\hat{A}_{I, S}^{\dagger}$ for two positive semidefinite matrices $S$ and $T$, then $\mathcal{R}(A T)=$ $\mathcal{R}(A S)$.

(ii) Similarly to the well-known formula $T T^{\dagger}=P_{T}$, we have that $A \hat{A}_{I, T}^{\dagger}=P_{A T}$.

(iii) If $A$ is a matrix in $\mathbb{R}^{m \times m}$ and $\hat{A}_{I, T}^{\dagger} A=A \hat{A}_{I, T}^{\dagger}$, then $P_{A T}\left(T^{\dagger}\right)^{\frac{1}{2}}=\left(T^{\dagger}\right)^{\frac{1}{2}} P_{T A^{*}}$.

Proof.

(i) Let the two positive semidefinite matrices $S, T$ such that $\hat{A}_{I, T}^{\dagger}=\hat{A}_{I, S}^{\dagger}$. Then $A \hat{A}_{I, T}^{\dagger}=A \hat{A}_{I, S}^{\dagger} \Rightarrow P_{A T}=P_{A S}$.

(ii) Trivial.

(iii) If $\hat{A}_{I, T}^{\dagger} A=A \hat{A}_{I, T}^{\dagger}$, then

$$
\begin{gathered}
\left(T^{\dagger}\right)^{\frac{1}{2}}\left(A\left(T^{\dagger}\right)^{\frac{1}{2}}\right)^{\dagger} A=A\left(T^{\dagger}\right)^{\frac{1}{2}}\left(A\left(T^{\dagger}\right)^{\frac{1}{2}}\right)^{\dagger} \Rightarrow \\
\left(T^{\dagger}\right)^{\frac{1}{2}}\left(A\left(T^{\dagger}\right)^{\frac{1}{2}}\right)^{\dagger} A\left(T^{\dagger}\right)^{\frac{1}{2}}=A\left(T^{\dagger}\right)^{\frac{1}{2}}\left(A\left(T^{\dagger}\right)^{\frac{1}{2}}\right)^{\dagger}\left(T^{\dagger}\right)^{\frac{1}{2}}
\end{gathered}
$$

and so,

$$
\left(T^{\dagger}\right)^{\frac{1}{2}} P_{\left(A\left(T^{\dagger}\right)^{\frac{1}{2}}\right)^{*}}=P_{A T}\left(T^{\dagger}\right)^{\frac{1}{2}}
$$

but, since $\mathcal{R}\left(\left(A\left(T^{\dagger}\right)^{\frac{1}{2}}\right)^{*}\right)=\mathcal{R}\left(T A^{*}\right)$ we have that $P_{A T}\left(T^{\dagger}\right)^{\frac{1}{2}}=\left(T^{\dagger}\right)^{\frac{1}{2}} P_{T A^{*}}$.

In the sequel, we present an example which clarifies Definition 3.3. In addition, the difference between the proposed minimization $\left(x \in \mathcal{N}(T)^{\perp}\right)$ and the minimization for all $x \in \mathcal{H}$ is clearly indicated.

ExAmple 3.6. Let $\mathcal{H}=\mathbb{R}^{4}$, the matrix $A=\left[\begin{array}{llll}1 & 2 & 1 & -1 \\ 0 & 1 & 0 & -1\end{array}\right]$ and the positive semidefinite matrix

$$
T=\left[\begin{array}{llll}
2 & 2 & 2 & 2 \\
2 & 3 & 3 & 3 \\
2 & 3 & 4 & 4 \\
2 & 3 & 4 & 4
\end{array}\right]
$$


Here, the equation is $A x=b$ with $b=\left[\begin{array}{l}2 \\ 1\end{array}\right]$.

Using Definition 3.3 we can compute the T-restricted weighted inverse $\hat{A}_{I_{2}, T}^{\dagger}$ :

$$
\hat{A}_{I_{2}, T}^{\dagger}=\left[\begin{array}{cc}
1.2667 & -1.8667 \\
-0.1333 & 0.9333 \\
-0.1333 & -0.0667 \\
-0.1333 & -0.0667
\end{array}\right]
$$

Then $\hat{A}_{I_{2}, T}^{\dagger} b=\left(\frac{2}{3}, \frac{2}{3},-\frac{1}{3},-\frac{1}{3}\right)^{T}$ is a minimal $T$ semi-norm least squares solution of $A x=b$, restricted on the range of $T$.

It is easy to see that all vectors $u \in \mathcal{R}(T)$ have the form $u=(x, y, z, z)^{T}, x, z \in \mathbb{R}$, so the solution has the expected form. With calculations we can find that all vectors belonging to $\mathcal{R}(T)$ and also satisfying $A u=b$ have the form of

$$
u=(-2 z, z+1, z, z)^{T}, z \in \mathbb{R} .
$$

In Figure 1, we plot the values of $z$ and the corresponding values of $\|u\|_{T}=u T u^{\prime}$. It is clear that the value of $z=-\frac{1}{3}$ gives the minimum value for the semi-norm $\|\cdot\|_{T}$. Therefore the vector $\hat{u}=\left(\frac{2}{3}, \frac{2}{3},-\frac{1}{3},-\frac{1}{3}\right)^{T}$ found from the T-restricted weighted inverse $\hat{A}_{I_{2}, T}^{\dagger}$ minimizes the semi-norm $\|\cdot\|_{T}$. In this case, $\|\hat{u}\|_{T}^{2}=1.333$.

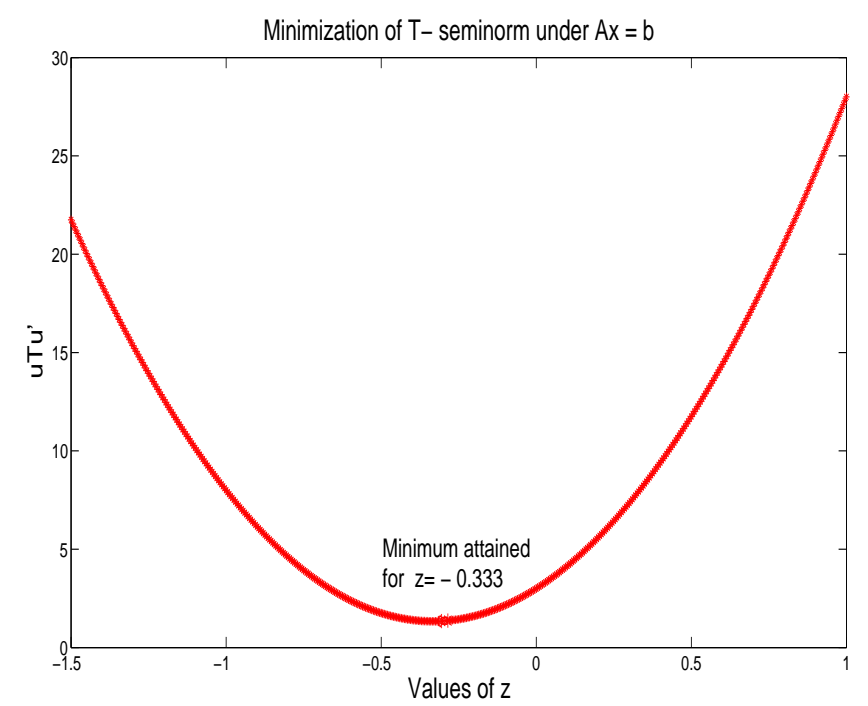

Fig. 3.1. Constrained minimization of $\|\cdot\|_{T}, u \in \mathcal{N}(T)^{\perp}$ under $A x=b$. 
If the minimization takes place for all vectors $u \in \mathbb{R}^{4}$, then the minimum seminorm vector satisfying $A u=b$ is the vector $w=(0,0,1,-1)^{T}$ and we have that $\|w\|_{T}=0$.

4. Relations with the $V$-orthogonal projector. For every matrix $X \in \mathbb{R}^{n \times p}$ and a positive semidefinite matrix $V \in \mathbb{R}^{n \times n}$, the matrix

$$
P_{X: V}=X\left(X^{*} V X\right)^{\dagger} X^{*} V
$$

is called the $V$-orthogonal projector with respect to the semi-norm $\|\cdot\|_{V}$ (see e.g., [21] or [22, Section 3]). The $V$-orthogonal projector is unique when $r(V X)=r(X)$. In this section, we study relations between $\hat{A}_{I, T}^{\dagger}$ and $P_{A: T}$. We will make use of the following Theorem:

TheOREM 4.1. ([21, Theorem 7]) Let $P_{X: V}$ be as given, and suppose $r(V X)=$ $r(X)$. Then $P_{X: V}=X\left(V^{\frac{1}{2}} X\right)^{\dagger} V^{\frac{1}{2}}$.

Using the above notation, we can see that similarly to the Moore-Penrose inverse property $T^{\dagger} T=P_{T^{*}}$ we have the following:

Proposition 4.2. Let $T \in \mathbb{R}^{m \times m}$ be a positive semidefinite matrix and $A \in$ $\mathbb{R}^{n \times m}$. If $r\left(T^{\dagger} A^{*}\right)=r\left(A^{*}\right)$, then the T-restricted weighted inverse $\hat{A}_{I, T}^{\dagger}$ has the property $\hat{A}_{I, T}^{\dagger} A=P_{A^{*}: T^{\dagger}}$.

Proof. As we can see, in our case we have that $X \equiv A^{*}$ and $V \equiv T^{\dagger}$. Therefore, $P_{A^{*}: T^{\dagger}}=A^{*}\left(\left(T^{\dagger}\right)^{\frac{1}{2}} A^{*}\right)^{\dagger}\left(T^{\dagger}\right)^{\frac{1}{2}}$. As such,

$$
P_{A^{*}: T^{\dagger}}^{*}=\left(T^{\dagger}\right)^{\frac{1}{2}}\left(A\left(T^{\dagger}\right)^{\frac{1}{2}}\right)^{\dagger} A=\hat{A}_{I, T}^{\dagger} A .
$$

REMARK 4.3. The relation $r\left(T^{\dagger} A^{*}\right)=r\left(A^{*}\right)$ can be replaced by

$$
\mathcal{N}(T) \cap \mathcal{N}(A)^{\perp}=\mathcal{N}(T) \cap \mathcal{R}\left(A^{*}\right)=\{0\} .
$$

Proof. Since $r\left(T^{\dagger} A^{*}\right)=r\left(A^{*}\right)-\operatorname{dim}\left(\mathcal{N}\left(T^{\dagger}\right) \cap \mathcal{R}\left(A^{*}\right)\right)$ we must have that $\mathcal{N}\left(T^{\dagger}\right) \cap$ $\mathcal{R}\left(A^{*}\right)=\{0\}$ but since $T$ is positive, $\mathcal{N}\left(T^{\dagger}\right)=\mathcal{N}(T)$. So, $r\left(T^{\dagger} A^{*}\right)=r\left(A^{*}\right)$ is equivalent to $\mathcal{N}(T) \cap \mathcal{R}\left(A^{*}\right)=\{0\}$.

By the above remark, we can have many results related to the $V$-orthogonal projector, using Theorems 7 and 8 in [21].

Proposition 4.4. Let $T \in \mathbb{R}^{m \times m}$ be a positive semidefinite matrix and $A \in$ $\mathbb{R}^{n \times m}$, such that $\mathcal{N}(T) \cap \mathcal{R}\left(A^{*}\right)=\{0\}$. Then the following hold:

(i) $A P_{A^{*}: T^{\dagger}}=P_{A T} A$. 
(ii) In the case when $A \hat{A}_{I, T}^{\dagger}=\hat{A}_{I, T}^{\dagger} A$, we have that $P_{A^{*}: T^{\dagger}}^{*}=P_{A T}$.

(iii) $\hat{A}_{I, T}^{\dagger} P_{A T} A=\hat{A}_{I, T}^{\dagger} A$.

(iv) The matrix $\hat{A}_{I, T}^{\dagger} A$ is hermitian.

(v) $\hat{A}_{I, T}^{\dagger} A=P_{T}$.

Proof.

(i) $A P_{A^{*}: T^{\dagger}}=A \hat{A}_{I, T}^{\dagger} A$ from Proposition 4.2 which is equal to $P_{A T} A$ from Proposition 3.4 .

(ii) If $A \hat{A}_{I, T}^{\dagger}=\hat{A}_{I, T}^{\dagger} A$, then from Proposition 4.2 and Proposition 3.5 we have $P_{A T}=P_{A^{*}: T^{\dagger}}^{*}$

(iii) From [21, Theorem 7], we have that $P_{A^{*}: T^{\dagger}}^{2}=P_{A^{*}: T^{\dagger}}$ and so $\left(\hat{A}_{I, T}^{\dagger} A\right)^{2}=$ $\left(\hat{A}_{I, T}^{\dagger} A\right) \Rightarrow \hat{A}_{I, T}^{\dagger} A \hat{A}_{I, T}^{\dagger} A=\hat{A}_{I, T}^{\dagger} A$. Therefore $\hat{A}_{I, T}^{\dagger} P_{A T} A=\hat{A}_{I, T}^{\dagger} A$.

(iv) From $\left[21\right.$, Theorem 8], we have that $P_{A^{*}: T^{\dagger}}=P_{A^{*}: T^{\dagger}}^{\prime}$ and so $\hat{A}_{I, T}^{\dagger} A$ is hermitian.

(v) From [21, Theorem 8], we have that $P_{A^{*}: T^{\dagger}}=P_{T^{\dagger}}=P_{T}$ and so $\hat{A}_{I, T}^{\dagger} A=$ $P_{T}$.

An important paper for the interested reader relating seminorms and generalized inverses is [18].

5. Numerical experiments. For the exhibition of our proposed method effectiveness, we have performed numerical experiments for the proposed constrained minimization problem, for both full and sparse positive semidefinite matrices. In particular, the present section is divided in two subsections, the first one gives numerical results of the proposed method for the case of non-sparse positive semidefinite matrices, and the second gives the corresponding results for the case of sparse positive semidefinite matrices. Also, for the purpose of monitoring the performance, we present tables with the execution times of the proposed MATLAB functions. All the numerical tasks have been performed by using the MATLAB R2009a environment on an Intel(R) Pentium(R) Dual CPU T23101.46 GHz 1.47 GHz 32-bit system with $2 \mathrm{~GB}$ of RAM memory running on the Windows Vista Home Premium Operating System.

5.1. Non-sparse positive semidefinite matrices. In this subsection, we discuss the case of non-sparse positive semidefinite matrices in order to clarify the efficiency of the proposed method. For the purpose of monitoring the performance, we present in Table 1 the execution times of the proposed method (wsol) on a set of non-sparse positive semidefinite matrices. In order to construct this set of matrices, we used a set of 9 singular test matrices of size $1000 \times 1000$ with a "large" condition number from Higham's Matrix Computation Toolbox (mctoolbox), see [10]. 
The proposed method was tested with a MATLAB function named wsol and the time responses have been recorded using a MATLAB function named testpsd. These two MATLAB functions can be found in [11], together with a help file and several MATLAB files that implement all the proposed examples from this paper. The interested reader is strongly encouraged to run these files under the guidance of the included help file in order to verify the experimental results of this paper.

TABLE 5.1

Non-sparse positive semidefinite matrices, PSD matrix size $1000 \times 1000$.

\begin{tabular}{ccc}
\hline Generator Matrix & PSD matrix rank & wsol(time in seconds) \\
\hline chow & 999 & 43.45 \\
cycol & 250 & 53.24 \\
gearmat & 999 & 52.52 \\
kahan & 168 & 55.19 \\
lotkin & 12 & 54.76 \\
prolate & 511 & 76.22 \\
hilb & 13 & 53.94 \\
magic & 3 & 58.79 \\
vand & 24 & 52.33
\end{tabular}

From Table 1, it is evident that the proposed numerical method, based on the introduction of the wsol function, enables us to perform fast estimations for a variety of dimensions. In fact, the joint amount of tests, calculations and further considerations required to reach the goal may well render the manual solution process a prohibiting task. By using the wsol function an interested user can solve the minimization problem within a few seconds. Note that the wsol function requires the presence of a MATLAB function in order to calculate the generalized inverse of a matrix. There are several methods for computing the Moore-Penrose inverse of a matrix. Some of the most commonly used methods are based on the Singular Value Decomposition method (MATLAB's pinv function), the conjugate Gram-Schmidt process and the Moore-Penrose inverse of partitioned matrices (see [23]), and iterative methods which are derived from the second Penrose equation (see [20]). In this work, for the determination of the Moore-Penrose inverse matrix, we use the results of a recent work, [12], where a very fast and reliable method is presented. This method is efficiently applicable in full or sparse matrices, ill-conditioned or not.

5.2. Sparse positive semidefinite matrices. In this subsection, we test the proposed method on sparse positive semidefinite matrices from the Matrix Market Repository [15]. We chose six matrices from the set BCSSTRUC1 (BCS Structural Engineering matrices, eigenvalue matrices), for no specific reason other than these 
matrices have the required properties, i.e., sparse and positive semidefinite. As in the previous section, we followed a similar reasoning in order to test the efficiency of the proposed method. For this purpose, the proposed method was tested with a MATLAB function named spwsol and the time efficiency was tested with the sptestm file. Note that all the necessary files that implement the proposed examples from the Matrix Market collection can be found in [11].

TABLE 5.2

Sparse positive semidefinite matrices.

\begin{tabular}{cccc}
\hline matrix name & size & structural rank & spwsol(time in seconds) \\
\hline BCSSTM01 & 48 & 24 & 0.0023 \\
BCSSTM03 & 112 & 72 & 0.0072 \\
BCSSTM04 & 132 & 66 & 0.0115 \\
BCSSTM05 & 153 & full & 0.016 \\
BCSSTM07 & 420 & full & 4.62 \\
BCSSTM10 & 1086 & full & 66.35 \\
\hline
\end{tabular}

From Table 2, it is evident that the proposed numerical method, based on the introduction of the spwsol function, enables us to perform fast estimations for a variety of matrix dimensions.

6. Concluding remarks. In this work, we define the $T$-restricted weighted generalized inverse of a singular matrix $A$ with respect to a positive semidefinite matrix $T$, which defines a seminorm for the space. We assume that $T$ is positive semidefinite, so the minimal seminorm solution is considered for all vectors belonging to $\mathcal{N}(T)^{\perp}$. Numerical experiments show that the proposed method performs well for both full and sparse positive semidefinite matrices.

Therefore, the proposed method can find applications also in many financial problems, apart from the usual matrix optimization areas such as statistical modeling, linear regression, electrical networks, filter design, etc.

Acknowledgment. The authors would like to thank two anonymous referees for their remarks and suggestions which improved this article significantly.

\section{REFERENCES}

[1] A. Ben-Israel and T.N.E. Grenville. Generalized Inverses: Theory and Applications. SpringerVerlag, Berlin, 2002.

[2] M. Broom. On the number of local maxima of a constrained quadratic form. Proc. Roy. Soc. London, Ser. A, 443(1919):573-584, 1993. 
[3] J. Cambell and A. Lo, and A.C. MacKinlay. The Econometrics of Financial Markets. Princeton University Press, 1997.

[4] S.L. Campbell and C.D. Meyer. Generalized Inverses of Linear Transformations. Dover Publications Inc., New York, 1991.

[5] W. La Cruz. Extension of the Barzilai- Borwein method for quadratic forms in finite Euclidean spaces. Numer. Funct. Anal. Optim., 30(3/4):306-321, 2009.

[6] L. Elden. Perturbation theory for the least squares problem with equality constraints. SIAM J. Numer. Anal., 17:338-350, 1980.

[7] L. Elden. A weighted pseudoinverse, generalized singular values, and constrained least squares problems. BIT, 22:487-502, 1982.

[8] C.W. Groetsch. Generalized Inverses of Linear Operators. Marcel Dekker Inc., New York, 1977.

[9] R. Manherz and S. Hakimi. The generalized inverse in network analysis and quadratic - error minimization problems. IEEE Trans. Circuit Theory, 16(4):559-562, 1969.

[10] N.J. Higham. The Matrix Computation Toolbox. Availiable at http://www maths.manchester . ac.uk/ higham/mctoolbox/.

[11] V.N. Katsikis. Personal webpage, http://faculty.teipir.gr/vkatsikis. Link: MATLAB files for research papers.

[12] V.N. Katsikis, D. Pappas, and A. Petralias. An improved method for the computation of the MoorePenrose inverse matrix. Appl. Math. Comput., 217:9828-9834, 2011.

[13] D. Luenberger. Optimization by Vector Space Methods. Wiley Publ., New York, 1969.

[14] H. Markowitz. Portfolio selection. J. Finance, 7(1):77-91, 1952.

[15] Matrix Market. National Institute of Standards and Technology, Gaithersburg, MD. Available at http://math.nist.gov/MatrixMarket/.

[16] N. Minamide and K. Nakamura. Minimum error control problem in Banach space. Technical Report 16, Nagoya University, Nagoya, Japan, 1969.

[17] N. Minamide and K. Nakamura. A restricted pseudoinverse and its applications to constrained minima. SIAM J. Appl. Math., 19:167-177, 1970.

[18] S.K. Mitra and C.R. Rao. Projections under seminorms and generalized Moore-Penrose inverses. Linear Algebra Appl., 9:155-167, 1974.

[19] D. Pappas. Minimization of constrained quadratic forms in Hilbert spaces. Ann. Funct. Anal., 2(1):1-12, 2011.

[20] M. Tasić, P. Stanimirović, and M. Petković. Symbolic computation of weighted Moore-Penrose inverse using partitioning method. Appl. Math. Comput., 189:615-640, 2007.

[21] Y. Tian and Y. Takane. On V-orthogonal projectors associated with a semi-norm. Ann. Inst. Stat. Math., 61:517-530, 2009.

[22] y. Tian and H. Wang. Characterizations of EP matrices and weighted-EP matrices. Linear Algebra Appl., 434:1295-1318, 2011.

[23] F. Toutounian and A. Ataei. A new method for computing Moore-Penrose inverse matrices. $J$. Comput. Appl. Math., 228:412-417, 2009. 